\title{
STUDIES OF COMPOSITION OF SURFACES AND INTERFACES WITH THE USE OF AUGER ELECTRON SPECTROSCOPY
}

\author{
S. Mróz \\ Institute of Experimental Physics, University of Wrocław \\ Pl. Maxa Borna 9, 50-204 Wrocław, Poland
}

Processes leading to the Auger electron emission from the sample bombarded with a primary electron beam are discussed. It is shown that every element has its characteristic spectrum of Auger lines, and that owing to a small inelastic mean free path of Auger electrons, information obtained from Auger electron spectroscopy concerns the composition of surface layer of 0.5-1 nm thick. Experimental methods of Auger electron spectroscopy are presented together with problems connected with separation of Auger electrons from the secondary electron spectrum. Advantages and disadvantages of some electron energy spectrometers are considered. Methods of quantitative Auger analysis of homogeneous samples with the use of standards and catalogues of Auger spectra are presented. The role of matrix corrections in quantitative Auger analysis is discussed. Problems arising in Auger analysis of insulating samples are considered and methods of discharging of such samples are presented. Depth profiling of inhomogeneous samples by Auger electron spectroscopy with ion bombardment sputtering is described, and possibilities and limitations of this procedure are discussed. Principles of scanning Auger microscopy are presented. Factors determining the lateral resolution are discussed. Possibilities of Auger electron spectroscopy and scanning Auger microscopy in analysis of composition of grain boundaries exposed by in situ fracture are presented and discussed. Possibilities and limitations of Auger electron spectroscopy in the investigation of solid state surfaces are summarized.

PACS numbers: $68.35 .-\mathrm{p}, 68.55 .-\mathrm{a}$

\section{Introduction}

In the last thirty years, electron spectroscopies (Auger electron spectroscopy (AES), X-ray photoelectron spectroscopy (XPS), ultraviolet photoelectron spectroscopy (UPS), electron energy loss spectroscopy (EELS)) occurred to be important tools in the investigation of composition and atomic and electronic structures of the solid state surface layer. Results of such investigations are widely exploited 
in practical applications in the material science because the properties of a surface layer with a thickness in the range of nanometer are extremally important for the quality of many products. Talking about the surface layer one has in mind the boundary between a solid state sample and surrounding atmosphere as well as interfaces between different layers in this sample and intergranular regions in polycrystalline samples.

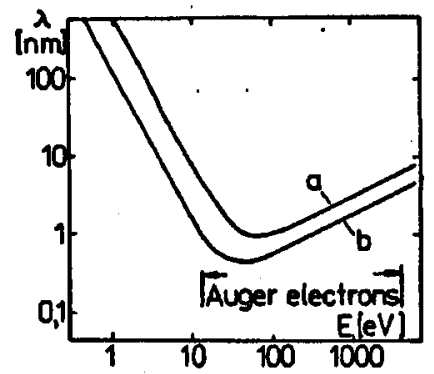

Fig. 1. Dependence of the inelastic mean free path of electrons on their energy. (a) for inorganic compounds, (b) - for elements.

Electron spectroscopies are especially suited to such investigations because the free path of electrons in a solid state is in the range of nanometer for electron energy of 10-1000 eV (see Fig. 1). Thus, information given by electron spectroscopies concerns just the above-mentioned surface layer. Among electron spectroscopies, the Auger electron spectroscopy (AES) is the simplest and very effective method applied in the material science.

\section{Physical foundations of Auger electron spectroscopy}

There is a lot of books and reviews where physical foundations of AES are widely presented and discussed (see, for example, Refs. [1-4]). The present work contains only some information about the Auger process.

When one of the deeper electron levels in an atom (see, for example, Fig. 2 for a silicon crystal) is ionized by an impact of the electron at this level with an

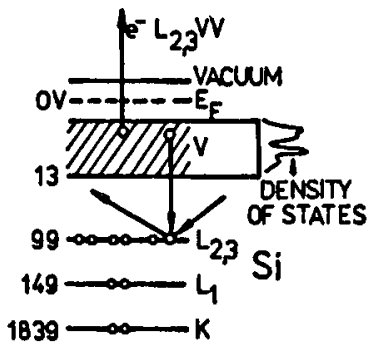

Fig. 2. A schema of the Auger process $\left(L_{2,3} V V\right.$ transition $)$ in silicon sample. 


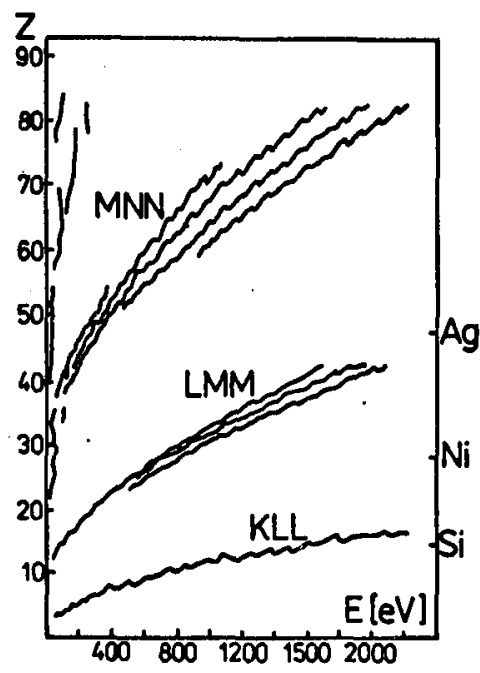

Fig. 3. A diagram of Auger electron energy dependence on the atomic number $Z$.

electron, ion or photon striking the sample from the outside, the vacant place at this level is quickly occupied by an electron from some higher level. The released energy can be emitted as an X-ray photon or transmitted to the next electron in the same atom, in the surrounding atom, or to the electron in the electron gas of the sample. Such an electron can be emitted from the sample and it is called an Auger electron. Energy of this electron is determined only by energies of electron levels in the emitting atom and is independent of the mechanism of the first ionization mentioned above. Because for the many-electron atom different electron transitions can be involved in the Auger electron emission, Auger electrons with different energies can be emitted from the atoms of a given element. Thus, every element is characterized by its own set of Auger electron energies (see Fig. 3) and can be identified in the sample by the analysis (Auger electron spectroscopy) of the energy spectrum of electrons emitted from this sample. Most of Auger electrons emitted from a depth larger than the inelastic mean free path lose a part of their energy before leaving the sample and are not detected as Auger electrons characteristic of a given element. Thus, information obtained from AES concerns only the surface layer of the sample investigated.

\section{Auger electron spectrometers}

Most often, the Auger electron emission is excited by bombardment the sample with an electron beam, because such a beam can be easily focused and scanned on the sample surface. To avoid scattering of primary electrons on gas molecules in the sample surroundings and to preserve the unchanged state of the sample surface, Auger electron spectrometer should be installed in an ultra-high vacuum system with a pressure as low as $10^{-7}-10^{-9} \mathrm{~Pa}$. For a proper preparation of the sample surface, systems for its heating and ion bombardment are necessary (see Fig. 4). The electron beam causes a secondary electron emission and the Auger 

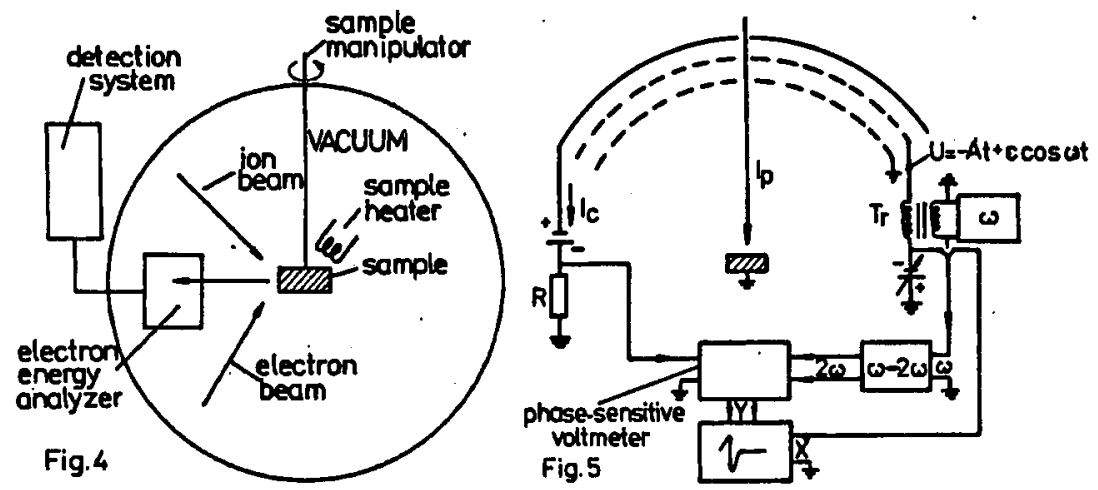

Fig. 4. Schematic view of the Auger electron spectrometer.

Fig. 5. An electric scheme of the Auger electron spectrometer with the retarding field analyzer.

electrons must be separated from a large background of secondary electrons. This can be done, when electrostatic electron energy analyzers have a modulation of the control voltage and a phase-sensitive detection of the signal. In a retarding field analyzer (RFA), which is a non-dispersive, high-pass electron energy filter, the saw-tooth control voltage applied to the second grid (see Fig. 5) is modulated by a small sinusoidal voltage with the frequency $\omega$ in the range of $1 \mathrm{kHz}$ while the phase-sensitive detector is tuned to the frequency of $2 \omega$. As a result, the signal of secondary electrons, which changes slowly with the change of electron energy in the energy distribution of all electrons emitted from the sample, is effectively attenuated while the Auger electrons are represented in the detected signal by the characteristic "Auger peak" (see Fig. 6b). The height of this peak is, roughly speaking, proportional to the Auger electron current.

Advantages of RFA are its simplicity, a large acceptance angle, insensitivity to the exact position of the sample, and the possibility to use this analyzer for observation of low energy electron diffraction (LEED) patterns as well. However, the large part of the whole secondary electron current reaches the detector of this analyzer. This leads to large noises of the detector current which limits the analyzer sensitivity.

In the so-called cylindrical mirror analyzer (CMA), trajectories of electrons emitted from the sample are bent in an electric field between two coaxial cylindrical electrodes (see Fig. 7) and electrons with a chosen energy (determined by the voltage applied to those electrodes) are directed to the collector while electrons with lower and higher energies are captured on electrodes. Owing to the proper geometry of the sample-entrance slit system, the second order focusing takes place in this analyzer, which gives simultaneously high transparency and good resolution. Auger spectrum of the nickel sample obtained with the use of CMA is shown in Fig. 6a. Because the CMA is a narrow-band electron energy filter, only a small part of secondary electrons reaches the collector and the noises of the detected signal are appreciably smaller than in RFA. Thus, the CMA is more sensitive and 


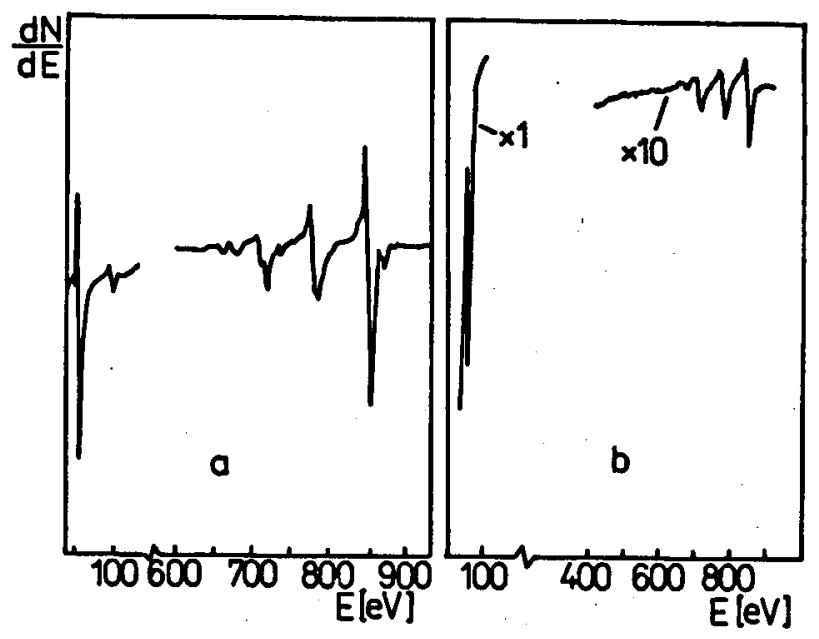

Fig. 6. Auger spectrum for nickel. (a) - obtained with a cylindrical mirror analyzer, (b) - obtained with a retarding field analyzer.

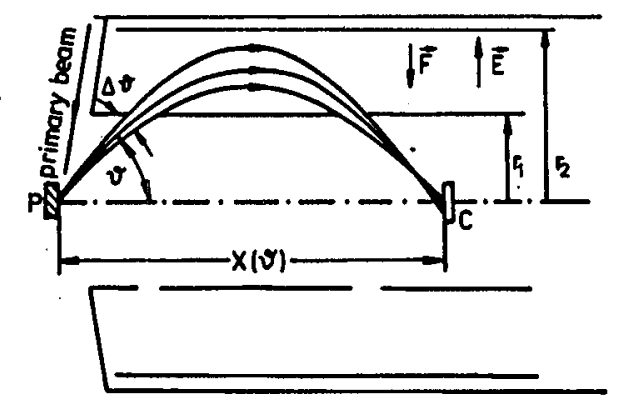

Fig. 7. A schematic view of a cylindrical mirror analyzer.

the analysis can be much faster (because a small time constant of the detector can be used). On the other hand, the acceptance angle of CMA is not as large as in RFA and its resolution is sensitive to the sample position. Because of its advantages, a CMA is the most commonly used in Auger electron spectrometers.

There are a number of other analyzers used in AES which are not discussed in the present paper. More information on RFA, CMA, and other analyzers can be found, for example, in Ref. [5].

\section{Quantitative Auger analysis of homogeneous samples}

For homogeneous samples, the Auger signal for the $i$-th element (most often the Auger peak height $h_{i}$ stands for it in AES) is proportional to the concentration $n_{i}$ of atoms of this element on the surface layer. Besides, this signal depends on the cross-section $\sigma$ for the ionization initiating the Auger process, probability $\gamma$ of the Auger transition after this ionization, backscattering factor $r$ taking into account the participation of electrons backscattered in the deeper layers of the 
sample in Auger electron emission excitation, inelastic mean free path $\lambda$ of Auger electron, and a current of primary electrons $I$

$$
h_{i}=A I \sigma \gamma r \lambda n_{i}=a_{i, M} I n_{i},
$$

where $A$ is a certain constant determined by the spectrometer geometry and by the relation between the Auger electron current and the corresponding Auger peak height. Indices in the constant $a_{i, M}$ indicate the $i$-th element contained in the sample (matrix) $M$.

Among the factors connecting $h_{i}$ and $n_{i}$ only the current $I$ can be exactly known, while the others are rather difficult to exact determination. For this reason, the quantitative Auger analysis (QAA) is, as a rule, performed with the use of standards of pure elements expected in the sample, which are placed in the spectrometer together with the sample or using standard spectra of pure elements taken from catalogues (for example Refs. [6-8]). When the Auger peak of a pure standard of the $i$-th element is considered, one can write

$$
h_{i, S}=a_{i, S} I n_{i, S} \text {. }
$$

Combining relations (1) and (2) one obtains

$$
n_{i}=n_{i, S}\left(a_{i, S} / a_{i, M}\right)\left(h_{i} / h_{i, S}\right) .
$$

If we are interested only in relative atomic concentration $C_{i, M}$ of particular elements in the sample, we can write

$$
C_{i, M}=\frac{n_{i}}{n_{M}}=\frac{n_{i}}{\sum_{j=1}^{N} n_{j}} .
$$

Applying relation (3) to all $n_{j}$ in Eq. (4) one obtains

$$
\begin{aligned}
C_{i, M} & =\left[\sum_{j=1}^{N}\left(\frac{n_{j, S}}{n_{i, S}}\right)\left(\frac{a_{j, S}}{a_{i, S}}\right)\left(\frac{a_{i, M}}{a_{j, M}}\right)\left(\frac{h_{i, S}}{h_{j, S}}\right)\left(\frac{h_{j}}{h_{i}}\right)\right]^{-1} \\
& =\left[\sum_{j=1}^{N} F_{i, j}\left(\frac{h_{i, S}}{h_{j, S}}\right)\left(\frac{h_{j}}{h_{i}}\right)\right]^{-1} .
\end{aligned}
$$

Now, we can take Auger peak heights $h_{j, S}$ for pure standards from the catalogues of Auger spectra, provided that the same type of analyzer and the same method of measurement were used by authors of the catalogue and in our measurements.

In the simplest approach to QAA (in QAA without matrix correction), one supposes that $F_{i, j}=1$ for all components which enables calculation of $C_{j, M}$ from relation (5). However, an error of such calculations can even be larger than $30 \%$. In the most sophisticated approach, all $F_{i, j}$ are calculated with the use of $n_{j, S}$ taken from proper tables and semiempirical or theoretical formulae for $\lambda$ and $r$ for Auger electrons emitted from the sample $M$ with a composition calculated using the simplest approach mentioned above and from the pure standards of particular elements. With the use of such procedure, an error can be as small as $2-3 \%$ in the case of many two-component metallic alloys.

It should be pointed out that, as it was shown in Ref. [9], almost the same accuracy can be obtained if relation (5) has a following form: 


$$
\begin{aligned}
C= & {\left[\sum_{j=1}^{N} F_{i, j}^{\prime}\left(\frac{n_{j, S}}{n_{i, S}}\right)\left(\frac{h_{i, S}}{h_{j, S}}\right)\left(\frac{h_{j}}{h_{i}}\right)\right]^{-1} } \\
& \cong\left[\sum_{j=1}^{N}\left(\frac{n_{j, S}}{n_{i, S}}\right)\left(\frac{h_{i, S}}{h_{j, S}}\right)\left(\frac{h_{j}}{h_{i}}\right)\right]^{-1},
\end{aligned}
$$

where one supposes that

$$
F_{i j}=\left(\frac{a_{j, S}}{a_{i, S}}\right)\left(\frac{a_{i, M}}{a_{j, M}}\right)=\left(\frac{a_{j, S}}{a_{j, M}}\right)\left(\frac{a_{i, M}}{a_{i, S}}\right)=1 .
$$

More information about QAA and the role of matrix corrections in it can be found in Ref. [4].

\section{Auger electron spectroscopy of insulators}

In analysis of insulating samples a problem arises with charging of the sample surface. This charging can be positive or negative, depending on the primary beam incidence angle and energy (because the secondary emission factor $\delta$ changes with electron incidence angle and energy in the manner shown schematically in Fig. 8) and can increase or decrease, respectively, the potential of the surface region bombarded with the electron beam. The potential changes up to the value for which the sum of the primary beam current, the current of secondary emission and current flowing through the sample is equal to zero.

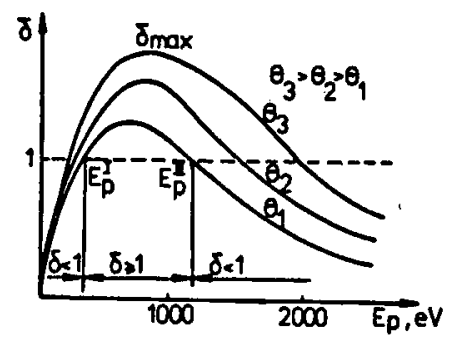

Fig. 8. Dependence of the secondary emission factor $\delta$ on the electron energy $E$ and the incident angle $\theta$ of the primary electron (measured with respect to the surface normal).

The change of the surface potential leads to the change of the kinetic energy of all emitted electrons in the entrance of the analyzer and, as a result, to the shift of the whole energy spectrum. Moreover, the primary beam can be moved, out of control, to the regions of the sample with a higher potential and trajectories of emitted electrons can be bent in the vicinity of the sample which influences the analyzer resolution.

All those effects are essentially reduced when the condition $\delta>1$ is satisfied (which can be frequently obtained by the choice of a proper electron energy and/or a large incidence angle of the primary beam). In such a case the increase in the sample potential is effectively compensated by low-energy secondary electrons attracted to the higher potential region. 
The other way to reduce the charging effects is to lower the resistivity of the sample. This can be done by diminution of the sample thickness and lateral dimensions, by proper doping the sample or by the increase in its temperature. In many cases it is enough to decrease the primary beam current or to increase the beam cross-section. A detailed discussion of this subject can be found in Ref. [10].

\section{Quantitative Auger analysis of heterogeneous samples}

As a matter of fact, the most important goal of QAA is to determine the distribution of concentration of particular elements in samples which are heterogeneous at distances comparable with the inelastic mean free path of Auger electrons. When the sample is heterogeneous only across the surface and not in depth and is composed of homogeneous regions of different composition, QAA can be performed with the use of methods presented above, provided that the electron beam diameter is appreciably smaller than dimensions of homogeneous regions and that this beam can be scanned across the sample surface with the possibility of recording the chosen Auger peak height for particular positions of the beam.

In a scanning Auger microscope (SAM), a map of composition of the sample surface is obtained in the above way. The resolution of such a map is limited by the primary electron beam diameter which can be easily diminished to the range of micrometer and, with the use of special, expensive electron guns, it can reach some tens of nanometer. Further decrease in the electron beam diameter is, in principle, possible but it does not lead to the improvement of the SAM resolution because the elastic and inelastic scattering of primary electrons lead to the creation of a region in the sample surface layer "filled" with those electrons which are able to excite the Auger electron emission. Because electron energies of $20-30 \mathrm{keV}$ are used in the case of sharply focused electron beams, one can expect that the diameter of the "filled" region is equal to ten or more nanometers (it is comparable with the inelastic mean free path of primary electrons). More detailed discussion of scanning Auger microscopy problems as well as examples of composition maps can be found, for example, in Ref. [11].

For samples heterogeneous in depth, the ion sputtering with the use of noble gas ions combined with simultaneous measurement of chosen Auger peak heights for some region in the flat part of the crater formed by this sputtering is the most frequently used method for obtaining the composition depth profile. The dependence of a given Auger peak height on the sputtering time (or on the sputtering ions dose) obtained in such an analysis has to be converted into the dependence of a given element concentration on the depth in the sample. To do this, the sputtering time should be converted into the depth and Auger peak height - into concentration.

Every depth profile obtained in such a way is broadened in comparison with the true one. To describe this broadening quantitatively, the depth resolution $\Delta z$ is introduced. Namely, when the sputtering goes through a very thin, delta function-like marker layer consisting of atoms of $i$-th element, one obtains the Gaussian function with its variation $\sigma$ as this element depth profile. The depth resolution $\Delta z$ is defined as $\Delta z=2 \sigma$. It is determined by inelastic mean free path of Auger electrons, by the roughness of the crater bottom induced by irregularities 
of the scattering process resulting from properties of the sample and the ion beam heterogeneity, and by possible changes in composition of the surface layer induced by the ion bombardment.

Assuming that different contributions $\Delta z_{j}$ to the measured $\Delta z$ are independent of each other, their superposition is statistically at random and one can write

$$
\Delta z=\left[\sum_{j}\left(\Delta z_{j}\right)^{2}\right]^{1 / 2} .
$$

For the part $\Delta z_{i}$ of $\Delta z$ connected with an inelastic mean free path $\lambda$ of Auger electrons one can use the relation $\Delta z=1.6 \lambda$, independently of the sputtering depth $z$. Other parts are constant or increase with increasing $z$. As a result, $\Delta z$ increases from a few nanometers at the beginning of the sputtering to tens of nanometer at the depth of several hundred nanometer.

More information about the depth profiling by the ion sputtering and about its optimum conditions can be found in Ref. [12].

To investigate grain boundary surfaces in polycrystalline samples such surfaces should be exposed to the primary electron beam by fracturing the sample in situ in an Auger spectrometer. To do this, a sample is prepared as a rod of a few millimeters in diameter with a notch at its mid-point. Such a sample is placed in the Auger spectrometer in the holder enabling its cooling to the temperature at which the fracture mainly along grain boundaries can be performed by a mechanical impact. The optimum sample temperature can vary for different samples from room temperature to liquid nitrogen one. The fracture should be performed under ultra-high vacuum conditions $\left(p \leq 10^{-7} \mathrm{~Pa}\right)$ and the time interval from the fracture to the Auger analysis should be as short as possible to avoid contamination of the freshly exposed surface with residual gases.

The surface exposed by such a fracture consists of intergranular and intragranular fragments with dimensions in the range of 10-100 $\mu \mathrm{m}$ and composition of particular fragments can differ essentially from one to other because of segregation of sample components to the grain boundaries. Thus, the analysis should be performed with the lateral resolution enabling distinction of particular fragments which can be easily achieved in a scanning Auger microscope. To avoid the diffusion of particular components from one fragment of the investigated surface to the other it is necessary in some cases to maintain the sample at low temperature for the whole time from the fracturing to the analysis.

The depth profile of particular fragments can be obtained by the ion sputtering of the whole sample surface.

More information about investigation of grain boundary surfaces together with representative examples can be found in Ref. [13].

\section{Possibilities and limitations of Auger electron spectroscopy}

Auger electron spectroscopy can give qualitative and quantitative information about presence and concentration of all elements with the atomic number $Z \geq 3$. Unfortunately, hydrogen and helium cannot be detected. The smallest detectable concentration depends on the type of electron energy analyzer used and 
is different for different elements and different Auger peaks. A typical limit of this sensitivity is in the range of $1 \%$ and depends on the time constant of the detection system. Thus, the AES is less sensitive than the methods developed in chemistry or optical spectral analysis. However, the unique property of AES is its sensitivity to the composition of the surface layer with a thickness as small as $1 \mathrm{~nm}$. Together with ion sputtering, AES gives the depth profile of particular element concentration for layers with thicknesses up to several hundred nanometers with resolution from a few to tens of nanometer.

With the use of a sharply focused primary electron beam scanned across the surface it is possible to obtain a map of concentration of a given element at this surface with the resolution reaching even tens of nanometer while combining such a scanning with the ion sputtering gives the three-dimensional picture of this concentration.

Some problems arise when insulating samples are analyzed but in the most cases effective methods of sample discharging can be found.

Primary electron beams can induce appreciable changes of the surface layer state (decomposition of molecules of complex samples and molecules adsorbed at the surface, electron stimulated desorption, heating of the small bombarded region). Samples sensitive to such effects should be treated with low-intense and defocused beams.

Auger spectrometers have to be installed in ultra-high vacuum systems. The location of the new sample in its place without opening the system to the atmosphere is a complicated task demanding additional, expensive elements of the vacuum system and especially designed sample holder. A much simpler solution of this problem is to open the system for each introduction of a new sample. However, the pumping procedure necessary for obtaining the ultra-high vacuum takes many hours.

Sophisticated Auger spectrometers enabling scanning Auger microscopy with the extremal resolution are very expensive and can be operated only by high-qualified users. However, a lot of very valuable information can be obtained with simpler systems which can be easily operated.

\section{Acknowledgments}

This work was supported by the University of Wroctaw under grant No. $2016 / \mathrm{W} / \mathrm{IFD} / 95$.

\section{References}

[1] A. Joshi, L.E. Davis, P.W. Palmberg, in: Melhods of Surface Analysis, Ed. A.W. Czanderna, Elsevier, Amsterdam 1975, p. 159.

[2] C.C. Chang, in: Characterization of Solid Surfaces, Eds. P.F. Kane, G. Larrabe, Plenum, New York 1974, p. 509.

[3] S. Mróz, Spektroskopia Elektronowa Augera, Wydawnictwo Uniwersytetu Wrocławskiego, Wrocław 1992 (in Polish).

[4] S. Mróz, Progress in Surface Science 46, 377 (1994).

[5] D. Roy, J.D. Carette, in: Electron Spectroscopy for Surface Analysis, in series Topics in Current Physics, Vol. 4, Ed. H. Ibach, Springer Verlag, Berlin 1977, p. 13. 
[6] P.W. Palmberg, G.E. Riach, R.E. Weber, N.C. Mac Donald, Handbook of Auger Electron Spectroscopy, Physical Electronics Inc., Edina, Minnesota 1972.

[7] L.E. Davis, N.C. MacDonald, P.W. Palmberg, G.E. Riach, R.E. Weber, Handbook of Auger Electron Spectroscopy, Physical Electronic Division, Perkin-Elmer Corp., Minnesota 1976.

[8] G.E. McGuire, Auger Electron Spectroscopy Reference Manual, Plenum Press, New York, London 1979.

[9] S. Mróz, W. Dolinski, Acta Phys. Pol. A 81, 201 (1992).

[10] S. Hofmann, J. Electron Spectr. Relat. Phenom. 59, 15 (1992).

[11] D.W. Harris, R.S. Nowicki, in: Auger and X-ray Photoelectron Spectroscopy, in series Practical Surface Analysis, Vol. 1, 2nd ed., Eds. D. Briggs, M.P. Seah, John Wiley \& Sons, Chichester 1994, p. 257.

[12] S. Hofmann, Progress in Surface Science 36, 35 (1991); S. Hofmann, in: Auger and $X$-ray Photoelectron Spectroscopy, in series Practical Surface Analysis, Vol. 1, 2nd ed., Eds. D. Briggs, M.P. Seah, John Wiley \& Sons, Chichester 1994, p. 143.

[13] M.P. Seah, in: Auger and X-ray Photoelectron Spectroscopy, in series Practical Surface Analysis, Vol. 1, 2nd ed., Eds. D. Briggs, M.P. Seah, John Wiley \& Sons, Chichester 1994, p. 311. 